Communication et organisation

$35 \mid 2009$

Repenser la communication dans les organisations publiques

\title{
Communication intercommunale : entre construction d'image du territoire et modification du comportement des usagers
}

David Huron et Grégory Spieth

\section{OpenEdition}

\section{Journals}

Édition électronique

URL : http://journals.openedition.org/communicationorganisation/764

DOI : 10.4000/communicationorganisation.764

ISSN : $1775-3546$

Éditeur

Presses universitaires de Bordeaux

Édition imprimée

Date de publication : 1 juin 2009

Pagination : 112-122

ISSN : 1168-5549

Référence électronique

David Huron et Grégory Spieth, « Communication intercommunale : entre construction d'image du territoire et modification du comportement des usagers », Communication et organisation [En ligne], 35 | 2009, mis en ligne le 01 décembre 2012, consulté le 19 avril 2019. URL : http:// journals.openedition.org/communicationorganisation/764; DOI : 10.4000/

communicationorganisation.764 
Dossier : Repenser la communication des organisations publiques

\title{
Résumé
}

Cet article a pour objet de s'interroger sur la nature des objectifs stratégiques d'un établissement public de coopération intercommunale et leur traduction en termes de communication. Il s'agira entre autres de préciser la place et le rôle de la participation des citoyens au niveau des actions de communication intercommunale. Pour illustrer le propos, une étude fondée sur des entretiens a été effectuée au sein de la CANCA (Communauté d'Agglomération de Nice Côte d'Azur). Les entretiens semi-directifs ont été effectués auprès du responsable " service collecte et gestion des déchets ménagers ", du responsable « ambassadeur du tri » et du directeur de la communication.

\section{Mots-clés}

Communication, Démocratie participative, Gouvernance, Identité du territoire, Intercommunalité.

\begin{abstract}
This article aims to examine the nature of the strategic objectives of Public Establishments of Inter-municipal Cooperation and their consequences in terms of communication. It needs to clarify the role of the citizens on the conception and the development of communication. A study based on interviews was conducted in the CANCA (Community of Agglomeration of Nice Cote d'Azur). The semistructured interviews were conducted in this Community with the responsible of the service collection and waste, the responsible of the collect selective information officers and the director of the communication.
\end{abstract}

Key-Words

Communication, Participatory Democracy, Governance, territory identity, Intercommunity.

\section{Auteurs}

David Huron est Maître de Conférences en Sciences de Gestion à l'Université de Nice-Sophia Antipolis. Il dirige le Master 2 Management public et l'IUP Management du sport de l'IAE de Nice.

Grégory Spieth est Attaché Temporaire d'Enseignement et de Recherche à l'IAE de Nice (Université de Nice Sophia Antipolis). Ses recherches en Sciences de Gestion sont centrées sur le management public. 


\title{
Communication intercommunale : entre construction d'image du territoire et modification du comportement des usagers
}

David Huron, Grégory Spieth

\author{
huron@unice.fr \\ spiethgregory@hotmail.com
}

Les lois de décentralisation de 1982 ont considérablement modifié la société française. Ainsi les collectivités territoriales sont devenues des acteurs publics dont l'importance se mesure par leur budget, les missions de service public qu'elles assurent, ou les investissements qu'elles effectuent. Les communes, premier niveau administratif, sont particulièrement nombreuses (territorialement construites sur la base des paroisses) et possèdent des profils hétérogènes. L'émergence et le développement de l'intercommunalité traduit la volonté de régler les difficultés de gestion des territoires et la recherche de cohérence du développement local.

Cette contribution a pour objet de s'interroger sur la nature des objectifs stratégiques d'un EPCI (établissement public de coopération intercommunale) et leur traduction en termes de communication. Il s'agira entre autres de préciser la place et le rôle de la participation des citoyens au niveau des actions de communication intercommunale. Pour illustrer le propos, une étude à base d'entretiens a été effectuée dans une communauté d'agglomération récemment créée, où l'intercommunalité intégrée n'existait pas avant les lois Chevènement de 1999. Le choix de l'EPCI a été conditionné par le fait que ni la structure, ni les habitants, n'ont bénéficié d'effets d'expérience communicationnels antérieurement. Ainsi, l'étude a porté sur les actions de communication en matière de tri sélectif au sein de la CANCA (Communauté d'Agglomération de Nice Côte d'Azur $^{118}$. Les entretiens semi-directifs ont été effectués au sein de cet EPCI auprès du responsable «service collecte et gestion des déchets ménagers », du responsable des « ambassadeurs du tri » et du responsable de la communication.

Après avoir souligné les enjeux de la communication intercommunale, il conviendra de développer les soubassements théoriques de ce type

${ }^{118}$ La CANCA est devenue depuis le $1^{\text {er }}$ janvier 2009, une communauté urbaine : Nice-Côte d'Azur 
de communication. Pour autant le statut des EPCI amène à procéder à une différenciation des comportements communicationnels par rapport à une municipalité ; le récepteur supposé n'est pas identique.

\section{Les enjeux de la communication intercommunale}

La loi du 12 juillet 1999 relative au renforcement et à la simplification de la coopération intercommunale a introduit et généralisé un nouvel échelon décisionnel administratif. L'émergence de ces EPCI intégrés tels que les communautés de communes (moins de 50000 habitants), les communautés d'agglomération (de 50000 à 500000 habitants) et les communautés urbaines ${ }^{119}$ (plus de 500000 habitants), a considérablement modifié le cadre organisationnel et communicationnel local. Dans certains cas, le périmètre de ces structures a été déterminé en fonction de logiques purement administratives sans qu'il y ait automatiquement de définition d'identité du territoire. En outre, à la différence des communes ou des départements (échelons administratifs qui entourent territorialement les EPCI), il n'y a pas d'élection au suffrage universel direct dans ces structures. Ces premiers éléments ont des conséquences directes sur les enjeux de la communication intercommunale. En premier lieu, la jeunesse de ces structures implique souvent une méconnaissance de leur existence ou un déficit d'identité. Or, le législateur, se substituant au marché, oblige les collectivités territoriales et leurs émanations à communiquer ${ }^{120}$. Les EPCI se trouvent donc dans une situation où ils doivent communiquer à la fois sur leurs missions, mais aussi sur leur identité auprès de publics multiples en fonction des options choisies (citoyens, touristes, entreprises, etc.). Cette recherche de reconnaissance et de construction d'image est altérée, en second lieu, par une difficulté à exister dans le paysage communicationnel local. En effet tous les échelons administratifs communiquent, parfois sur des sujets identiques. Dans de nombreux cas, le positionnement communicationnel de l'EPCI par rapport à la ville centre n'est pas clairement annoncé, ce qui implique au mieux une absence d'identification de la structure intercommunale, au pire un amalgame avec la ville centre.

\footnotetext{
${ }^{119}$ Les communautés de communes existent depuis 1992 et les communautés urbaines depuis 1966. La loi du 12 juillet 1999 (loi Chevènement) consacre leur existence.

${ }^{120}$ La loi du 12 avril 2000, relative aux droits des citoyens est venue s'ajouter aux dispositions de la loi du 12 juillet 1999 relative au renforcement et à la simplification de la coopération intercommunale.
} 
La communication appliquée aux EPCI peut revêtir des formes diverses en fonction du degré d'implication des publics cibles. Le niveau minimal observé est celui de la transmission de l'information (journaux, publicité, etc.). Cet état ne prend pas en compte les éventuelles réactions des citoyens et illustre une conception descendante et hiérarchique de la communication. L'introduction d'une rétroaction dans la démarche de communication constitue un début de co-construction dans le processus de décision (enquêtes, sondages, etc.). Ces dispositions déjà présentes et obligatoires dans les collectivités territoriales sous l'impulsion du législateur ${ }^{121}$, peuvent permettre de corriger des actions menées par l'EPCI ${ }^{122}$. L'émergence de l'interactivité augmente la capacité à co-construire une décision (Huron, Spindler, 2008). Ainsi, la généralisation des Technologies de l'Information et de la Communication dans les EPCI a parfois permis, via des outils tels que les espaces de concertation numérique (chats et forums) de récolter plus rapidement de l'information, comme par exemple les réactions à des projets. La récente émergence de débats portant sur la démocratie participative locale traduit l'intérêt pour la co-construction de la décision entre élus, citoyens et autres acteurs locaux. Pourtant, si ce questionnement est opportun pour les collectivités territoriales, on peut s'interroger sur son application dans les EPCI, compte tenu de l'absence de rapport élus-électeurs. Par ailleurs, les objectifs de communication des EPCI (cognitifs, affectifs et conatifs), sont les mêmes que ceux recherchés dans l'entreprise, dans les collectivités territoriales ou dans d'autres structures. La pondération des objectifs communicationnels et les choix en matière de communication traduisent les objectifs stratégiques fixés par l'EPCI.

\section{Entre l'agir communicationnel et la gouvernance démocratique}

Les modes de gouvernement de la démocratie locale peuvent se décliner en fonction de plusieurs aspects tels que le type de management et de régulation choisi, le type de conception de la démocratie, la nature de la citoyenneté retenue, etc. (Assens, Phanuel, 2001). Ainsi, les modes autocratique (la population n'est pas sollicitée), consultatif (la population est peu sollicitée) et réticulaire (sollicitation des habitants), illustrent bien les choix qu'ont à effectuer les responsables des EPCI. Ainsi, le concept de gouvernance prend

\footnotetext{
${ }^{121}$ Loi du 13 août 2004 relative aux libertés et aux responsabilités locales.

${ }^{122} \mathrm{La}$ loi du 28 février 2002 relative à la démocratie de proximité a rendu obligatoire la création de commissions consultatives des services publics locaux pour les EPCI de plus de 50000 habitants.
} 
toute sa place dans une organisation lorsque la logique gouvernementale a été dépassée. L'un des premiers éléments visibles illustrant la gouvernance est la participation de la population au processus de décision. Certaines démarches conduites dans des EPCI il y a quelques années (Communauté de Creusot-Montceau) montrent qu'il est possible de bâtir une relation de proximité avec les habitants et que cette dernière est un élément essentiel de la gouvernance d'agglomération (Contis, Dressayre, Philippart, 2004). Pour autant, l'approche théorique de la gouvernance est multiple et Paul Hirst (2000) par exemple, en dénombre cinq versions. D'autres auteurs estiment qu'il existe un véritable continuum coercitif entre le gouvernement et le citoyen, le partenariat se situant au milieu de ce continuum (Vigoda, 2002).

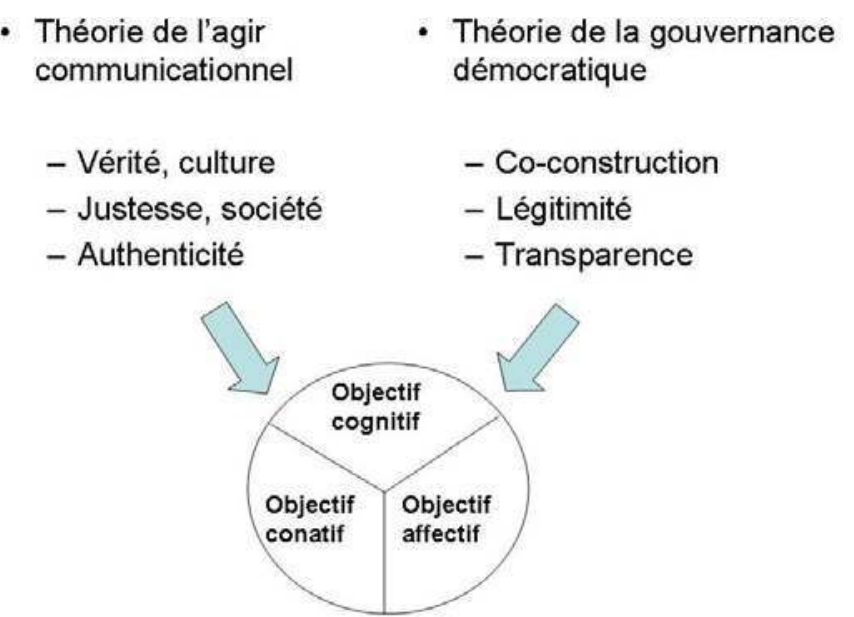

Figure 1 - Deux théories pour bâtir la communication intercommunale

La communication intercommunale, à l'image de la communication municipale, peut donc a priori prendre des formes différentes en fonction du type de relation à la décision. Deux théories illustrent cette dichotomie (figure 1): la théorie de l'agir communicationnel (Habermas, 1981) et la théorie de la gouvernance démocratique (Held, 1995). Les deux corpus théoriques proposent un triple éclairage autour de la prise de décision publique. En premier lieu, l'agir communicationnel pose la question de la vérité et de la culture. En d'autres termes, locuteurs et interlocuteurs sont-ils en accord sur l'analyse d'une situation ou sur la reconnaissance des faits? La théorie de la gouvernance démocratique aborde ce sujet sous l'angle de la co-construction de la décision. En deuxième lieu, la théorie de l'agir communicationnel met en évidence le rôle de la justesse et de la 
société. Ainsi, il convient d'apporter une réponse sur l'existence d'un accord sur les normes sociales au nom desquelles l'action peut être menée. La théorie de la gouvernance démocratique traite ces éléments sous l'angle de la recherche de légitimité de la décision effectivement prise. Enfin, l'authenticité constitue la troisième composante de l'agir communicationnel. Les interlocuteurs expriment-ils ce qu'ils pensent ou modifient-ils dans le discours leur pensée profonde ? La théorie de la gouvernance démocratique émet parallèlement comme objectif stratégique la recherche de la transparence. Cette transparence est d'ailleurs devenue règle pour le législateur.

Comme le souligne Jürgen Habermas (1991), la communication de la sphère publique s'accompagne de la manifestation de l'opinion publique. Afin de ne pas créer de déséquilibre, l'opinion publique est souvent prise en compte en amont dans le cadre de la conception de la communication à destination de la population. Dans la mesure où il n'existe pas pour l'instant d'élections au suffrage universel direct des représentants d'EPCI, le contenu et la construction de l'opinion publique ne semblent pas constituer une variable prioritaire dans l'élaboration de la communication intercommunale.

\section{Le tri sélectif à la CANCA : une communication tournée vers les administrés}

Lors de leur création, les communautés d'agglomération doivent choisir trois missions facultatives au minimum parmi cinq possibles. La CANCA a choisi de retenir les cinq missions dont l'assainissement. Cette volonté, qui traduit la stratégie d'un territoire, est identifiable par une communication entre autre tournée vers la collecte sélective. L'enjeu clairement affiché est de modifier les comportements des habitants en matière de tri des déchets ménagers. L'étude menée au sein de la CANCA a permis de souligner une constante (cf. annexe). La transmission d'un maximum d'informations techniques et pratiques sur la collecte sélective à destination des usagers constitue une priorité. Comme le soulignent certains auteurs, l'image et le sens affectés à l'action confèrent une visibilité et une lisibilité à l'institution (Fourdin, Poinclou, 2000 ; Garraud, 1990). Ainsi, l'information tente de répondre à l'objectif de changement de comportement attendu des citoyens en matière de collecte sélective. Le choix du support de communication le plus représentatif corrobore cette idée. En effet, le tract, sous des applications multiples, reprend les informations techniques et pratiques essentielles.

A partir du moment où la communication est assimilable à de l'information, la pratique de la participation est réduite à sa plus 
simple expression. Les enquêtés définissent l'habitant comme «absent » dans le pilotage des actions de communication publiques. Ils s'accordent à dire que c'est une démarche épisodique plus « consultative» qui pourra être envisagée dans le futur. Cette absence de prise en compte des finalités de la participation montre qu'il n'existe pas de co-construction dans la décision publique, ce qui militerait pour une utopie de la démocratie participative dans les structures intercommunales. L'application des deux théories précédemment évoquées pour expliquer la construction de la communication intercommunale trouve donc ici ses limites. Les raisons de cette limite sont essentiellement imputables à la nature même de l'EPCI intégré (établissement jeune, absence de suffrage universel direct, etc.), car la co-construction de la décision publique est observable dans les communes.

Les collectivités territoriales entretiennent des relations avec leurs administrés sur le thème d'un vécu communicable sur leur territoire ${ }^{123}$. Cette logique nécessite de raisonner sur un discours de la collectivité territoriale ancré dans le réel, ayant pour conséquence la construction d'une communication envisagée au coup par coup. Pour que la relation soit acceptable pour les parties prenantes, l'écart entre les promesses communicatives faites par la collectivité et le niveau d'attente des citoyens ne doit pas faire l'objet d'un décalage (Hetzel, 1999). Pour posséder des informations sur le ressenti de la population, il est nécessaire de mettre en œuvre une démarche évaluative concernant la stratégie de communication choisie. Cette démarche, couramment appliquée dans les municipalités, semble rencontrer des difficultés à s'imposer dans les EPCI. En effet, l'évaluation n'a pas de place clairement définie dans la conduite des actions de communication en matière de collecte sélective. L'idée même d'évaluation est mal comprise et peu soutenue par les acteurs. Avant d'être comprise comme une opportunité ou un besoin, l'évaluation est perçue comme une pratique plus ou moins imposée. Ainsi, les acteurs ont une représentation consensuelle de ce qui concerne l'absence des procédures possibles pour évaluer les actions de communication en matière de collecte sélective : "On ne fait pas le lien entre les actions de communication et leur impact sur le terrain ». La communication concernant la collecte sélective semble donc se développer aléatoirement sans vision stratégique co-construite avec la population.

${ }^{123}$ « Le territoire vécu réfère à une appartenance. Il est une identité engagée vis-à-vis d'un territoire, lequel se définissant [...] par la communauté qui l'habite et l'investit de sa subjectivité » (Caillouette, 2009, p.9). 
De surcroît, le choix de l'externalisation de la conception, du développement et de la mise en œuvre des campagnes de communication à une entreprise privée, traduit une certaine distance dans l'engagement de l'EPCI sur ce sujet. La conception d'un cahier des charges relativement succinct démontre le manque de vision stratégique en matière de communication sur la collecte sélective. L'entreprise délégataire, axée sur le fond de la campagne, n'impulsera pas de démarche évaluative si cette dernière n'est pas demandée et limitera l'interactivité avec la population.

La communication intercommunale possède ses propres spécificités et la tentative d'appliquer les modèles théoriques existant dans les municipalités semble pour l'instant atteindre ses limites. Le profil des EPCI joue bien entendu un rôle primordial. L'absence d'élection au suffrage universel direct implique que le niveau intercommunal privilégie plus l'administré que le citoyen (Kerrouche, 2004). Ce constat se traduit par une communication à caractère informatif, non co-construite où l'évaluation ne constitue pas un élément de pilotage. De même, le manque de légitimité démocratique des EPCI pousse ces derniers à limiter la communication identitaire, ce qui ne plaide pas en faveur d'une reconnaissance rapide de cet échelon par la population (Cohen-Bacrie, Lamarque, 2006). Les récents débats parlementaires semblent mettre en évidence les problèmes liés à cette absence de reconnaissance démocratique. Dans l'hypothèse où les EPCI intégrés feraient l'objet d'une élection au suffrage universel direct, des changements de stratégies de communication sont à prévoir. L'enjeu est de savoir si les EPCI garderont leur spécificité communicative ou s'ils se rapprocheront des modèles développés dans les communes.

\section{Bibliographie}

BARDIN N., L'analyse de contenu, Paris : PUF, 1996.

CAILlOUETTE J., "Appartenance, identité et territoire vécu: des notions centrales au développement des communautés ", In Revue Développement Social. 2009, vol. 9, n 3, pp. 9-10.

COHEN-BACRIE B., LAMARQUE P. Pratique(s) de la communication intercommunale, bilans et perspectives. Paris: Weka, 2006, $153 \mathrm{p}$.

ASSENS C., PHANUEL D., Les modes de gouvernement de la démocratie locale. In : LE DUFF R., RIGAL J.-J., SCHMIDT G. Eds. Démocratie et management local, Paris : Dalloz, 2001, pp. 49-68.

CONTIS L., DRESSAYRE P., PHILIPPART E., Intercommunalité et citoyenneté: quelles voies pour une proximité entre les structures 
Dossier : Repenser la communication des organisations publiques

intercommunales et les acteurs territoriaux? L'exemple des communautés urbaines en France. In : LE DUFF R., RIGAL J.-J. Eds. Démocratie et management local, Paris : Dalloz, 2004, pp. 183-193.

FOURDIN M., POINCLOU J.-B., «Le local au miroir de la communication intercommunale », In Hermès, 2000, $\mathrm{n}^{\circ}$ 26-27, pp. 283-294.

GARRAUD P., Discours des maires et construction locale du politique. Mots, 1990, n²5, pp. 7-20.

HABERMAS J., Théorie de l'agir communicationnel, Paris: Fayard, 1981.

HABERMAS J., The structural transformation of the public sphere, Cambridge, MA: The MIT Press, 1991, $301 \mathrm{p}$.

HELD D., Democracy and the Global Order: From the Modern State to Cosmopolitan Governance. Stanford: Stanford University Press, 1995, $324 \mathrm{p}$.

HETZEL P., Le marketing des villes : concept éphémère à la mode ou tendance de fond pour repenser la relation entre élus et citoyens?, In : LE DUFF R., RIGAL J.-J., Eds. Maire, entrepreneurs, emploi. Paris : Dalloz, 1999, pp. 318-326.

HIRST P., Democracy and Governance, In: PIERRE J., Debating Governance. Oxford: Oxford University Press, 2000, pp.13-35.

HURON D., SPINDLER J., «L'évaluation des politiques publiques locales : la régulation par le citoyen?», In Le management public en mutation, Paris: L'Harmattan, 2008, pp. 291-306.

KERROUCHE E., "Le lien manquant. Quelques réflexions sur les problèmes démocratiques des intercommunalités à la française », In : LE DUFF R., RIGAL J.-J., Eds. Démocratie et management local, Paris : Dalloz, 2004, pp. 371-392.

VIGODA E., From responsiveness to collaboration: Governance, citizens, and the next generation of public administration. Public Administration Review. 2002, vol. 62, $\mathrm{n}^{\circ}$ 5, pp. 525-540.

\section{Annexe : synthèse de l'analyse thématique des entretiens}

L'analyse thématique est définie par Bardin (1996, p. 77) comme « le comptage d'un ou plusieurs thèmes ou items de signification dans une unité de codage préalablement déterminée ». L'unité de codage est ici déterminée par la phrase. Le but de ce dénombrement thématique est de faire ressortir les opinions émises et de faire émerger le discours des professionnels. L'importance à accorder à ces thèmes se mesure alors en fréquence d'apparition (colonne «item»). Pour cette recherche, le choix a été fait de procéder à un encodage rapide après 
chaque entretien, pour parvenir à une meilleure compréhension du phénomène étudié. Ces constatations sont notées selon les critères présentés dans le tableau suivant.

\begin{tabular}{|c|c|c|c|}
\hline Thèmes & Indicateurs & Citations & Item \\
\hline \multirow{5}{*}{$\begin{array}{l}\text { Outils de } \\
\text { communication }\end{array}$} & $\begin{array}{l}\text { Les tracts } \\
\text { distribués dans les } \\
\text { boites aux lettres }\end{array}$ & $\begin{array}{l}\text { « Nous utilisons, beaucoup les } \\
\text { tracts à vocation informative } \\
\text { qui contiennent les horaires, les } \\
\text { modalités techniques de la } \\
\text { collecte sélective. » }\end{array}$ & 12 \\
\hline & Place des TIC & $\begin{array}{l}\text { «On a le site internet qui sert } \\
\text { pour mettre un maximum } \\
\text { d'information. » } \\
\text { "On commence à travailler sur } \\
\text { le mailing, on contacte quand } \\
\text { même } 20000 \text { personnes. » } \\
\text { « Les gens s'expriment peu sur } \\
\text { le site internet. » }\end{array}$ & 3 \\
\hline & $\begin{array}{l}\text { Utilisation de } \\
\text { Campagnes } \\
\text { medias, spot } \\
\text { radio. }\end{array}$ & $\begin{array}{l}\text { «Les campagnes radio sont } \\
\text { souvent utilisées, les annonces } \\
\text { de presse plus ponctuelles. » }\end{array}$ & 6 \\
\hline & $\begin{array}{l}\text { Animation } \\
\text { scolaire }\end{array}$ & $\begin{array}{l}\text { «Il faut savoir que le public } \\
\text { scolaire représente une des } \\
\text { meilleures cibles, puisque les } \\
\text { enfants d'aujourd'hui feront les } \\
\text { adultes de demain. » } \\
\text { «Tout au long de l'année, les } \\
\text { ambassadeurs du tri font des } \\
\text { animations scolaires. » }\end{array}$ & 8 \\
\hline & $\begin{array}{l}\text { Communication } \\
\text { de proximité }\end{array}$ & $\begin{array}{l}\text { «Les ambassadeurs du tri, tous } \\
\text { les mercredis passent dans les } \\
\text { quartiers pour sensibiliser les } \\
\text { citoyens à la collecte } \\
\text { sélective. » }\end{array}$ & 6 \\
\hline \multirow{2}{*}{$\begin{array}{ll}\text { Elaboration } & \text { de } \\
\text { campagne } & \text { de } \\
\text { presse } & \end{array}$} & $\begin{array}{l}\text { Externalisation de } \\
\text { la communication }\end{array}$ & $\begin{array}{l}\text { «On a une agence de } \\
\text { communication qui élabore les } \\
\text { projets sur la base de nos } \\
\text { besoins. » }\end{array}$ & 15 \\
\hline & $\begin{array}{l}\text { Cible de la } \\
\text { communication }\end{array}$ & $\begin{array}{l}\text { "Apporter la possibilité à tous } \\
\text { les habitants de trier quels que } \\
\text { soient leurs modes de } \\
\text { collecte ». }\end{array}$ & 7 \\
\hline
\end{tabular}


Dossier : Repenser la communication des organisations publiques

\begin{tabular}{|c|c|c|c|}
\hline \multirow{4}{*}{$\begin{array}{l}\text { Création } \\
\text { identité et } \\
\text { conduite du } \\
\text { changement } \\
\text { Identification } \\
\text { des objectifs de } \\
\text { communication }\end{array}$} & $\begin{array}{l}\text { Changement de } \\
\text { comportement }\end{array}$ & $\begin{array}{l}\text { "Objectif commun de ces } \\
\text { outils : faire trier les gens en } \\
\text { grande quantité. " } \\
\text { "Obtenir l'adhésion d'un } \\
\text { maximum de personne. » } \\
\text { " Notre but est de faire changer } \\
\text { les mauvais comportements et } \\
\text { d'introduire de nouveaux } \\
\text { comportements. » }\end{array}$ & 14 \\
\hline & $\begin{array}{l}\text { Faire connaître } \\
\text { ses missions }\end{array}$ & $\begin{array}{l}\text { " Les gens connaissent la mairie } \\
\text { et c'est tout, globalement les } \\
\text { français connaissent mal leurs } \\
\text { institutions » } \\
\text { " Nous connaissons un manque } \\
\text { de reconnaissance sur le } \\
\text { territoire. » }\end{array}$ & 3 \\
\hline & $\begin{array}{l}\text { Information des } \\
\text { publics }\end{array}$ & $\begin{array}{l}\text { "On n'informe pas les gens sur } \\
\text { la CANCA, on les informe sur } \\
\text { les déchets et les horaires. » }\end{array}$ & 8 \\
\hline & $\begin{array}{l}\text { Augmenter les } \\
\text { ressources }\end{array}$ & $\begin{array}{l}\text { "Plus les gens trient, plus on } \\
\text { collecte et plus on est } \\
\text { subventionné. » }\end{array}$ & 4 \\
\hline \multirow[t]{2}{*}{$\begin{array}{l}\text { Evaluation de la } \\
\text { communication } \\
\text { Modalités } \\
\text { d'évaluation }\end{array}$} & $\begin{array}{l}\text { Outils } \\
\text { d'évaluation des } \\
\text { actions de } \\
\text { communication }\end{array}$ & $\begin{array}{l}\text { «On n'a pas de bilan } \\
\text { d'évaluation des actions de } \\
\text { communication, on utilise tous } \\
\text { les modes de communication. } \\
\text { Après, tout dépend du budget. » } \\
\text { "On n'utilise pas d'outils } \\
\text { d'évaluation. » } \\
\text { "On ne fait pas le lien entre les } \\
\text { actions de communication et } \\
\text { leur impact sur le terrain. » }\end{array}$ & 12 \\
\hline & $\begin{array}{l}\text { Participation des } \\
\text { citoyens }\end{array}$ & $\begin{array}{l}\text { «Si un usager nous contacte, } \\
\text { c'est par l'utilisation du site } \\
\text { Internet et l'information } \\
\text { transmise est peu } \\
\text { constructive. » } \\
\text { «C'est un peu difficile } \\
\text { d'imaginer sa place [le public] } \\
\text { dans la communication.» }\end{array}$ & 6 \\
\hline
\end{tabular}

